\title{
Clinical Stage I Gastric Cancer AJCC v8
}

National Cancer Institute

\section{Source}

National Cancer Institute. Clinical Stage I Gastric Cancer A/CC v8. NCI Thesaurus. Code C133648.

Stage I includes: (T1, N0, M0); (T2, N0, M0). T1: T umor invades the lamina propria, muscularis mucosae, or submucosa. T2: Tumor invades the muscularis propria. N0: No regional lymph node metastasis. M0: No distant metastasis. (AJCC 8th ed.) 